\title{
The Canagliflozin and Renal Endpoints in Diabetes with Established Nephropathy Clinical Evaluation (CREDENCE) Study Rationale, Design, and Baseline Characteristics
}

\author{
Meg J. Jardine ${ }^{a, b}$ Kenneth W. Mahaffey ${ }^{c}$ Bruce Neal ${ }^{a}$, $-f$ Rajiv Agarwal ${ }^{g}$ \\ George L. Bakris $^{h}$ Barry M. Brenner ${ }^{i}$ Scott Bull ${ }^{j}$ Christopher P. Cannonk \\ David M. Charytan' Dick de Zeeuw ${ }^{m}$ Robert Edwards ${ }^{j}$ Tom Greene ${ }^{n}$ \\ Hiddo J.L. Heerspink ${ }^{m}$ Adeera Levin $^{\circ}$ Carol Pollock $^{p}$ David C. Wheeler $^{q}$ \\ John Xie ${ }^{j}$ Hong Zhang ${ }^{r}$ Bernard Zinman ${ }^{s}$ Mehul Desai ${ }^{j}$ Vlado Perkovic ${ }^{a}$ \\ on behalf of the CREDENCE study investigators
}

\begin{abstract}
${ }^{a}$ The George Institute for Global Health, UNSW Sydney, Sydney, NSW, Australia; ${ }^{b}$ Concord Repatriation General Hospital and University of Sydney, Sydney, NSW, Australia; 'Stanford Center for Clinical Research, Department of Medicine, Stanford University School of Medicine, Stanford, CA, USA; ${ }^{\mathrm{d}}$ The Charles Perkins Centre, University of

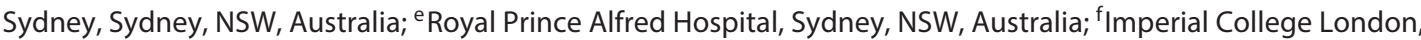
London, UK; ${ }^{9}$ Indiana University School of Medicine and VA Medical Center, Indianapolis, IN, USA; h University of Chicago Medicine, Chicago, IL, USA; 'Renal Division and Department of Medicine, Brigham and Women's Hospital, Harvard Medical School, Boston, MA, USA; jJanssen Research \& Development, LLC, Raritan, NJ, USA; ${ }^{k}$ Cardiovascular Division, Brigham and Women's Hospital and Baim Institute for Clinical Research, Boston, MA, USA; 'Renal Division,

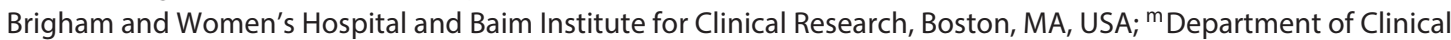
Pharmacy and Pharmacology, University of Groningen, University Medical Center Groningen, Groningen, The

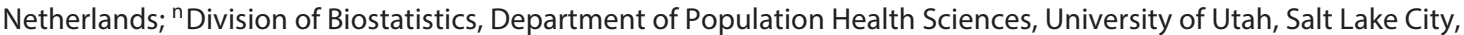
UT, USA; ${ }^{\circ}$ Division of Nephrology, University of British Columbia, Vancouver, BC, Canada; ${ }^{\mathrm{P}}$ Kolling Institute of Medical Research, Sydney Medical School, University of Sydney, Royal North Shore Hospital, St Leonards, NSW, Australia; ${ }^{9}$ Centre for Nephrology, UCL Medical School, London, UK; ${ }^{r}$ Renal Division of Peking University First Hospital, Beijing, China; ${ }^{5}$ Lunenfeld-Tanenbaum Research Institute, Mt Sinai Hospital, University of Toronto, Toronto, ON, Canada
\end{abstract}

\section{Keywords}

Diabetic nephropathy · Renal outcomes · Canagliflozin ·

Chronic kidney disease

\section{Abstract \\ Background: People with diabetes and kidney disease have a high risk of cardiovascular events and progression of kid- ney disease. Sodium glucose co-transporter 2 inhibitors low-}

er plasma glucose by reducing the uptake of filtered glucose in the kidney tubule, leading to increased urinary glucose excretion. They have been repeatedly shown to induce modest natriuresis and reduce $\mathrm{HbA} 1 \mathrm{c}$, blood pressure, weight, and albuminuria in patients with type 2 diabetes. However, the effects of these agents on kidney and cardiovascular

For all the site investigators in the CREDENCE trial, see www.karger.com/doi/10.1159/000484633.

\section{KARGER}

(C) 2017 S. Karger AG, Basel

E-Mail karger@karger.com

www.karger.com/ajn
Meg J. Jardine, MD, PhD, FRACP

Head, Renal Trials, George Clinical

The George Institute for Global Health, 1 King Street

Newtown NSW 2042 (Australia)

E-Mail mjardine@georgeinstitute.org.au 
events have not been extensively studied in patients with type 2 diabetes and established kidney disease. Methods: The Canagliflozin and Renal Endpoints in Diabetes with Established Nephropathy Clinical Evaluation (CREDENCE) trial aims to compare the efficacy and safety of canagliflozin versus placebo at preventing clinically important kidney and cardiovascular outcomes in patients with diabetes and established kidney disease. CREDENCE is a randomized, double-blind, event-driven, placebo-controlled trial set in in 34 countries with a projected duration of $\sim 5.5$ years and enrolling 4,401 adults with type 2 diabetes, estimated glomerular filtration rate $\geq 30$ to $<90 \mathrm{~mL} / \mathrm{min} / 1.73 \mathrm{~m}^{2}$, and albuminuria (urinary albumin:creatinine ratio $>300$ to $\leq 5,000 \mathrm{mg} / \mathrm{g}$ ). The study has $90 \%$ power to detect a $20 \%$ reduction in the risk of the primary outcome $(a=0.05)$, the composite of end-stage kidney disease, doubling of serum creatinine, and renal or cardiovascular death. Conclusion: CREDENCE will provide definitive evidence about the effects of canagliflozin on renal (and cardiovascular) outcomes in patients with type 2 diabetes and established kidney disease. Trial Registration: EudraCT number: 2013-004494-28; ClinicalTrials.gov identifier: NCT02065791.

(c) 2017 S. Karger AG, Basel

\section{Background and Rationale}

It is estimated that around the world there are currently more than 3 million people with chronic kidney disease $(\mathrm{CKD})$ requiring renal replacement therapy, and that this number will increase to more than 5 million by 2030 [1]. The most common cause of CKD in most countries is type 2 diabetes mellitus (T2DM). The estimated global prevalence of $\mathrm{CKD}$ due to diabetes is more than 100 million [2], and much of the expected increase in kidney failure is due to the rapid global increase in the prevalence of T2DM [3]. People affected by CKD have a reduced life expectancy and lower quality of life, as well as a substantially increased risk of cardiovascular disease and other adverse health outcomes [4]. The high cost of dialysis challenges health service budgets in both developing and developed countries. Development of treatments that can prevent or delay progression of CKD in patients with diabetes is a research priority at both an individual and a societal level.

Renin-angiotensin-aldosterone system blockade is currently the only treatment proven to prevent the decline of kidney function [5] and development of kidney failure [6] in T2DM, based primarily on the results of 2 parallel trials completed more than 15 years ago. While these agents are clearly effective in people with diabetes and established CKD, there is a high residual risk of deteriorating kidney function and other clinically important outcomes such as cardiovascular disease and death related to cardiovascular or kidney causes, even when the maximum labeled or maximally tolerated dose of these agents is used. Additional effective therapies are therefore urgently required.

Canagliflozin is a sodium glucose co-transporter 2 (SGLT2) inhibitor that blocks glucose reabsorption in the proximal tubule, causing glucosuria and natriuresis. In patients with T2DM, canagliflozin has been shown to improve glycemic control, lower blood pressure (BP), reduce body weight, and lower albuminuria compared with placebo or standard care targeting glycemic control [7]. Canagliflozin is also generally well tolerated with increased incidence of adverse events (AEs) related to the mechanism of SGLT2 inhibition (e.g., genital mycotic infections, osmotic diuresis-related AEs) [8]. There have been reports of diabetic ketoacidosis [9], fractures [10], amputations [11], and acute kidney injury [12] with canagliflozin; however, the incidence of these events was generally low across groups in clinical trials [8]. A recent study has shown evidence of stabilization in kidney function with canagliflozin compared to the sulfonylurea glimepiride, despite similar glycemic control [13]. These data suggest that canagliflozin may protect against the progression of CKD in T2DM. This hypothesis is also supported by recent secondary analyses from 2 cardiovascular outcome trials in the class showing that canagliflozin and empagliflozin stabilized kidney function and reduced the risk of adverse kidney outcomes as compared to placebo in populations defined by cardiovascular disease presence and risk [1416]. The use of SGLT2 inhibitors is therefore a promising treatment for the prevention of kidney and cardiovascular outcomes in people with diabetes and established nephropathy, but their efficacy remains to be confirmed in appropriately powered, randomized controlled trials in this population.

\section{Objectives}

The Canagliflozin and Renal Events in Diabetes with Established Nephropathy Clinical Evaluation (CREDENCE; ClinicalTrials.gov identifier: NCT02065791) study is designed to formally test whether canagliflozin reduces the risk of kidney failure and cardiovascular events in patients with T2DM and markers of established kidney disease compared to placebo when used in addition to standard of care. 


\section{Methods}

CREDENCE is a randomized, double-blind, placebo-controlled, parallel-group, multicenter clinical trial.

\section{Study Setting}

The study is being conducted in 695 sites in 34 countries in North America, Latin America, Europe, South Africa, and Asia Pacific (full list of countries and sites available in online suppl. Appendix 1; for all online suppl. material, see www.karger.com/ doi/10.1159/000484633).

\section{Eligibility Criteria}

The inclusion and exclusion criteria were designed to include a broad range of patients with diabetes at risk of progression of kidney disease. Potential patients are eligible if they are aged $\geq 30$ years and have a clinical diagnosis of T2DM with an HbA1c level of $\geq 6.5$ to $\leq 12.0 \%$ (except Germany, where the HbAlc range is $\geq 6.5$ to $\leq 10.5 \%)$. Patients are required to have kidney disease in the setting of T2DM in the absence of an alternative diagnosis to account for kidney pathology, although a clinical diagnosis is accepted without the requirement for a confirmatory kidney biopsy, in keeping with common clinical practice. Patients are required to have an estimated glomerular filtration rate (eGFR) $\geq 30$ to $<90 \mathrm{~mL} / \mathrm{min} / 1.73$ $\mathrm{m}^{2}$ and albuminuria (urine albumin:creatinine ratio [UACR] $>300$ to $\leq 5,000 \mathrm{mg} / \mathrm{g}$ [33.9-565.6 $\mathrm{mg} / \mathrm{mmol}]$ ). Patients believed to have nondiabetic kidney disease who have a history of treatment of kidney disease with immunosuppression, or a history of treatment with dialysis or kidney transplantation are excluded. In order to ensure that the study is able to assess the impact of canagliflozin on the progression of CKD, the intent is that approximately $60 \%$ of the patient population have stage $3 \mathrm{CKD}$ (rather than stage $2 \mathrm{CKD}$ ), with an eGFR of $\geq 30$ to $<60 \mathrm{~mL} / \mathrm{min} / 1.73 \mathrm{~m}^{2}$ at study entry.

All patients are required to be established on the maximum labeled or tolerated dose of an angiotensin-converting enzyme inhibitor (ACEi) or angiotensin receptor blocker (ARB) for $\geq 4$ weeks prior to randomization. Combination use of an ACEi and ARB or use of a direct renin inhibitor is not allowed. Patients were also excluded if they had concomitant use of an SGLT2 inhibitor within 12 weeks of randomization, current or past participation in another canagliflozin study, or known allergy, hypersensitivity, or intolerance to canagliflozin. In addition to the kidney-related exclusion criterion, patients with a history of cardiovascular events within the previous 12 weeks or a history of New York Heart Association class IV heart failure at any time are also excluded. Full exclusion criteria are summarized in online supplementary Appendix 2.

Patients are eligible if they fulfill the requirements of the inclusion and exclusion criteria at the screening visit and until the randomization visit.

\section{Screening and Run-In}

Patients are prescreened on the basis of eGFR and UACR by medical chart review ( 6 months retrospective) or by prospective laboratory assessment to identify those with eGFR $\geq 30$ to $<90 \mathrm{~mL} /$ $\mathrm{min} / 1.73 \mathrm{~m}^{2}$ and UACR $>300 \mathrm{mg} / \mathrm{g}$ ( $\left.>33.9 \mathrm{mg} / \mathrm{mmol}\right)$. In countries where UACR is not routinely measured as per standard of care, one of the following measures is substituted: albumin excretion rate $>300 \mathrm{mg} / 24 \mathrm{~h}$, urine protein:creatinine ratio $>500 \mathrm{mg} / \mathrm{g}$ $(>56.5 \mathrm{mg} / \mathrm{mmol})$, or protein excretion rate $>500 \mathrm{mg} / 24 \mathrm{~h}$. All po- tential patients who meet eligibility criteria at the screening visit may progress to the screening period. If eligibility is confirmed at the screening visit, patients are asked to undergo a single-blind placebo run-in period. The screening period, including the 2 -week, single-blind, placebo run-in period, can be up to 10 weeks to allow sufficient time for the optimization of care according to local guidelines. The primary purpose of the run-in period is to exclude prior to randomization those patients unlikely to adhere to the long-term treatment and follow-up regimen required by the trial. Patients who fail to take $\geq 80 \%$ of the scheduled run-in treatment are deemed ineligible.

During the screening period, a disqualifying laboratory test may be repeated once at investigator discretion when there is clinical reason. Patients failing entry criteria on physical or laboratory criteria are eligible for rescreening after appropriate clinical management at the discretion of the investigator and with the concurrence of the sponsor's medical monitor.

\section{Randomization}

Randomization is performed centrally through an interactive web response system using a computer-generated randomization schedule prepared by the study sponsor. Patients are randomly assigned in a 1:1 ratio to either canagliflozin $100 \mathrm{mg}$ or matching placebo using randomly permuted blocks with stratification by baseline eGFR category ( $\geq 30$ to $<45, \geq 45$ to $<60$, and $\geq 60$ to $<90$ $\mathrm{mL} / \mathrm{min} / 1.73 \mathrm{~m}^{2}$ ).

\section{Intervention and Control}

Canagliflozin $100 \mathrm{mg}$ was selected for this study due to its more favorable benefit to risk profile in the Phase 3 clinical development program compared with canagliflozin $300 \mathrm{mg}$, which demonstrated an increased risk of specific AEs (e.g., volume depletion AEs) in patients with CKD. Study treatment is provided in identical bottles of over-encapsulated canagliflozin $100 \mathrm{mg}$ tablets or placebo. $\mathrm{Pa}$ tients and all study staff will remain blinded to individual treatment allocation until the completion of the study. Patients take their study treatment once daily before the first meal of the day, with a log maintained with information of study capsule counts.

Study drug administration will continue until the completion of the study or until the patient experiences an event that requires permanent cessation of the study drug. Permanent cessation is required for the following reasons: patient commences dialysis for end-stage kidney disease (ESKD), receives a renal transplant, becomes pregnant, experiences biochemically confirmed diabetic ketoacidosis, or is a recipient of ongoing disallowed therapy. Temporary interruption is required for subjects who develop conditions that are associated with amputation, such as a critical limb ischemia, until the condition has resolved. Restarting study medication after interruption is encouraged after careful consideration of the benefits and risks associated with treatment.

Patients experiencing a doubling of serum creatinine or nonfatal cardiovascular events are recommended to continue study treatment and complete assessments at all scheduled visits. Study drug treatment may be temporarily interrupted for safety and/or tolerability reasons (such as hospitalizations for major surgical procedure or serious medical illness). The reasons for these interruptions are recorded if they last longer than 7 days. Patients who discontinue study treatment are requested to continue follow-up as originally planned whenever possible, or to allow abbreviated follow-up if the original schedule is no longer suitable. 


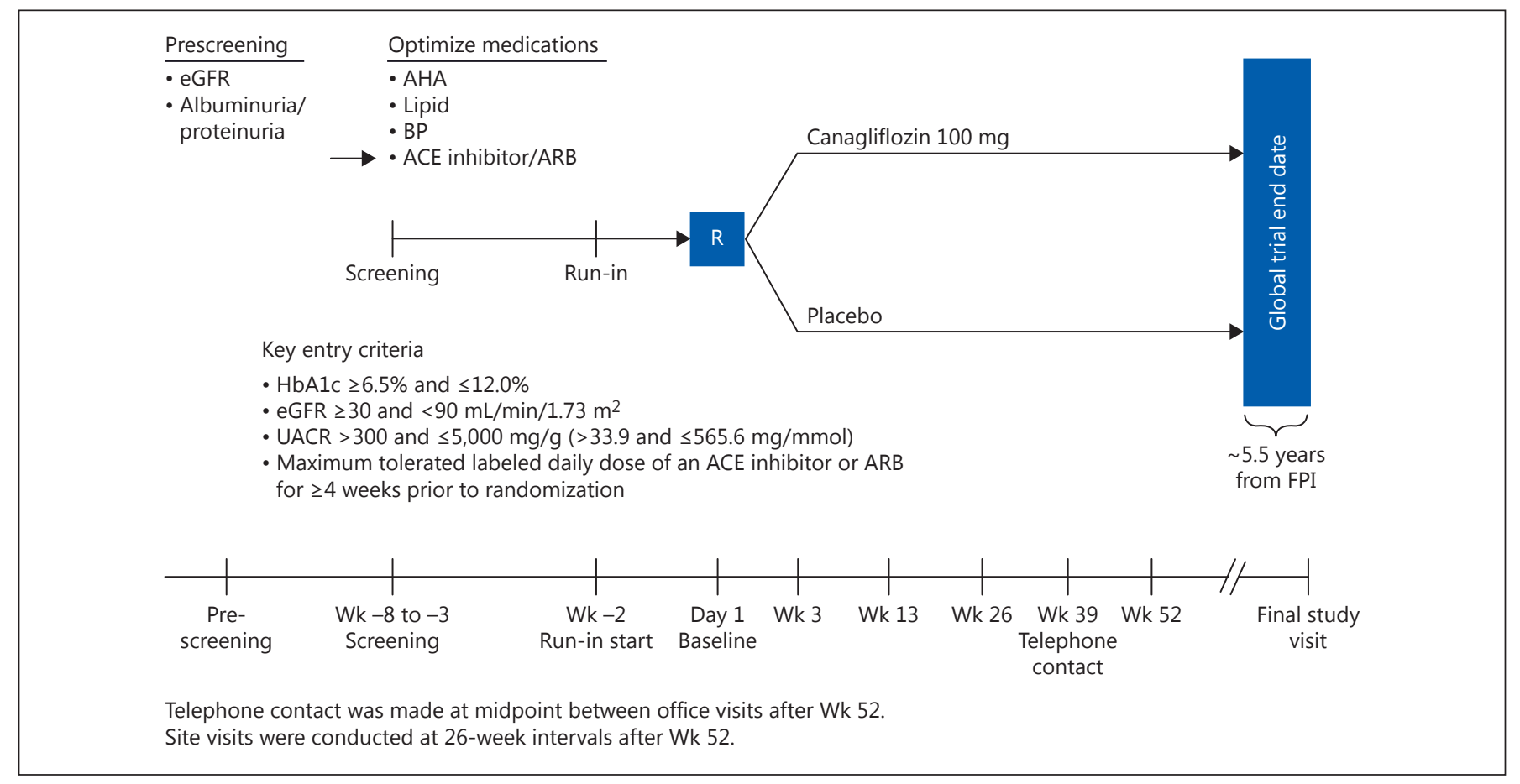

Fig. 1. Study design. eGFR, estimated glomerular filtration rate; AHA, anti-hyperglycemic agent; BP, blood pressure; ACE, angiotensinconverting enzyme; ARB, angiotensin receptor blocker; HbA1c, glycated hemoglobin; R, randomization; FPI, first patient in; Wk, week; UACR, urinary albumin:creatinine ratio.

\section{Background Care}

Glycemic control is reinforced with diet and exercise counseling, as well as instruction in the performance of self-monitored fasting blood glucose assessments according to standard local guidelines and recorded in a study diary. Glycemic management is otherwise left to the discretion of the responsible physician in line with applicable local guidelines. Likewise, the use of all other therapies is according to best practices followed throughout the course of the study and instituted according to local guidelines and policies. Consistent with the inclusion criteria, the combination use of an ACEi and ARB or use of a direct renin inhibitor is not allowed at any point during the study.

Patients receive counseling on their renal and cardiovascular risk factor medication. Investigators review the management of the patient's lipid control and BP prior to randomization and throughout the study in light of local guidelines. Sites are informed approximately biannually of the proportion of patients in their country/region and globally who are meeting glycemic control treatment targets, who reach treatment targets for systolic BP, and who are being treated with cardioprotective agents.

\section{Follow-Up Schedule}

Postrandomization follow-up in the first 12 months is scheduled at 3, 13, 26, 39, and 52 weeks after randomization and thereafter at 3-month intervals (Fig. 1). The visit at 39 weeks and every second visit thereafter are by telephone. Unscheduled blood tests or other safety assessment(s) can be collected at any time point. Patients will receive an additional telephone visit 30 days after the last dose of study drug. Patients who prematurely discontinue study treatment are encouraged to return for regular assessments to ensure full ascertainment of study outcomes and to support an intention-to-treat analysis for all outcomes.

\section{Outcomes and Endpoint Adjudication}

The primary endpoint is the composite of ESKD, doubling of serum creatinine, and renal or cardiovascular death (non-dialyzed). The secondary and exploratory endpoints are listed in Table 1 , and the definitions of the individual endpoint components are listed in online supplementary Appendix 3.

All renal and cardiovascular events that are components in the primary and secondary composite endpoints of the study (e.g., ESKD, doubling of serum creatinine, death) will be adjudicated by an Endpoint Adjudication Committee that is blinded to treatment assignment. The Endpoint Adjudication Committee will also be responsible for adjudicating suspected endpoint events of myocardial infarction (MI), stroke, hospitalized unstable angina, and hospitalized congestive heart failure.

Kidney function will be assessed by calculating the eGFR using the CKD-EPI (CKD Epidemiology Collaboration) formula. The baseline average serum creatinine value is calculated as the average of 2 pretreatment values collected up to 4 weeks apart. Serum creatinine will be measured at baseline; weeks 3, 13, and 26; and every 6 months thereafter. Doubling of serum creatinine from baseline will be confirmed by a central laboratory value at least 30 days (and preferably within 60 days) from the first doubling from baseline 
Table 1. Prespecified efficacy and safety endpoints and evaluations of the CREDENCE study

Primary and secondary efficacy endpoints

\begin{tabular}{ll}
\hline Primary endpoint & Composite of ESKD, doubling of serum creatinine, and renal or cardiovascular death \\
\hline Secondary & Composite of cardiovascular death and hospitalized congestive heart failure \\
endpoints & Cardiovascular death \\
& All-cause death \\
& Renal composite endpoint of ESKD, doubling of serum creatinine, and renal death \\
& Cardiovascular composite endpoint of cardiovascular death, nonfatal MI, nonfatal stroke, hospitalized congestive \\
heart failure, and hospitalized unstable angina
\end{tabular}

Safety evaluations and outcomes

\begin{tabular}{ll}
\hline Adverse events & $\begin{array}{l}\text { All adverse events will be collected and coded using the MedDRA from randomization until } 30 \text { days after the last } \\
\text { date of blinded study medication }\end{array}$ \\
\hline $\begin{array}{l}\text { Adverse events of } \\
\text { interest }\end{array}$ & $\begin{array}{l}\text { All malignancies, fatal pancreatitis, hemorrhagic/necrotising pancreatitis, severe hypersensitivity reactions (e.g., } \\
\text { angioedema, anaphylaxis, Stevens-Johnson syndrome), photosensitivity reactions, serious adverse events of hepatic } \\
\text { injury, nephrotoxicity/acute kidney injury, venous thromboembolic events, fractures, diabetic ketoacidosis (and } \\
\text { related adverse events including ketoacidosis, metabolic acidosis, or acidosis), amputation, and pregnancy }\end{array}$ \\
\hline Hypoglycemia & $\begin{array}{l}\text { All episodes of hypoglycemia (both symptomatic and asymptomatic) are recorded on a } \\
\text { dedicated hypoglycemia eCRF }\end{array}$ \\
\hline Safety laboratory tests & Chemistry, hematology, urinalysis \\
\hline Physical examination & Pulse, BP, weight \\
\hline
\end{tabular}

CREDENCE, Canagliflozin and Renal Events in Diabetes with Established Nephropathy Clinical Evaluation; ESKD, end-stage kidney disease; MI, myocardial infarction; eGFR, estimated glomerular filtration rate; BP, blood pressure; MedDRA, Medical Dictionary for Regulatory Activities.

detected on either local or central laboratory determinations. The use of medications known to impact serum creatinine levels (e.g., nonsteroidal anti-inflammatory drugs, trimethoprim, cimetidine, probenecid, aminoglycosides, amphotericin, ketoconazole, and clofibrate) is discouraged during the screening period and for approximately 2 weeks before any serum chemistry measurement during the course of the study.

To minimize intra-subject variability, first-morning-void urine specimens will be used, but collection is deferred during acute illness with fever, and strenuous exercise is to be avoided within 72 hours of a planned study visit. Due to the potential risk of unblinding that may occur if urine glucose levels are reported, investigators have been asked to obtain all post-baseline urinalyses through the central laboratory and not by a local laboratory, to avoid performing local dipstick urinalysis unless required for urgent medi- cal management (such as of diabetic ketoacidosis or other safety concerns), and to restrict local investigations to microscopy (rather than urinalysis) when microscopy is sufficient.

In addition to the main study analyses, patients are invited to contribute optional fasting plasma, serum, and urine samples to allow for potential exploratory biomarker analyses. When local regulations permit, patients are also invited to contribute an optional blood sample for pharmacogenomics testing. These samples will be stored and will be accessed to address research questions to be determined upon study completion.

AEs and serious AEs are being collected in accordance with good clinical practice and standard regulatory requirements. AEs will be coded using the Medical Dictionary for Regulatory Activities dictionary. During the study, AEs will be reported in conformity with regulatory requirements worldwide to ensure appropriate re- 
porting of safety information. Prespecified AEs of interest requiring collection of supplemental clinical parameters include all malignancies, fatal pancreatitis, hemorrhagic/necrotizing pancreatitis, severe hypersensitivity reactions (e.g., angioedema, anaphylaxis, StevensJohnson syndrome), photosensitivity reactions, hepatic injury, nephrotoxicity/acute kidney injury, venous thromboembolic events, fractures, diabetic ketoacidosis, amputation, and pregnancy.

\section{Statistical Analyses}

The impact of the intervention on the primary endpoint will be assessed by an intention-to-treat analysis using a Cox proportional hazard model with an indicator variable for the treatment as the predictor variable and stratification of the baseline hazard by pretreatment eGFR ( $\geq 30$ to $<45, \geq 45$ to $<60, \geq 60$ to $<90 \mathrm{~mL}$ / $\mathrm{min} / 1.73 \mathrm{~m}^{2}$ ). The estimated treatment effect will be expressed as the hazard ratio and its 95\% CI. Ratios of cause-specific hazards between the treatment groups will be obtained for each component of the primary efficacy composite endpoint (ESKD, doubling of serum creatinine, and renal or cardiovascular death), with stratification of the baseline hazard by the pretreatment eGFR group as in the analysis of the primary efficacy composite endpoint.

The secondary outcomes will be analyzed in a similar fashion as that of the primary analysis. If superiority of canagliflozin 100 mg over placebo for reducing the risk of the primary efficacy endpoint is established, the treatment effects in secondary endpoints will be tested subsequently in the following hierarchical order: (i) the composite endpoint of cardiovascular death and hospitalized congestive heart failure; (ii) the composite endpoint of cardiovascular death, nonfatal MI, and nonfatal stroke (i.e., 3-point MACE); (iii) hospitalized congestive heart failure; (iv) the renal composite endpoint of ESKD, doubling of serum creatinine, and renal death; (v) cardiovascular death; (vi) all-cause death; and (vii) the cardiovascular composite endpoint of cardiovascular death, nonfatal MI, nonfatal stroke, hospitalized congestive heart failure, and hospitalized unstable angina. Statistical significance is required before testing the next hypothesis in the hierarchical test procedure.

For on-treatment eGFR measurements, a linear mixed-effects model will be fitted to eGFR as a dependent variable, including treatment, stratification factor, baseline eGFR value, time (as a continuous variable), and treatment time interaction as fixed effects, and including subject effect as a random intercept and time as a random slope. The parameter of interest is the coefficient for treatment and time interaction term, which measures the slope difference between canagliflozin and placebo.

Safety analyses will be conducted on all patients who receive $\geq 1$ dose of double-blind study medication.

\section{Powering and Sample Size}

CREDENCE is an event-driven study. With a planned recruitment of at least 4,200 patients, it will have $90 \%$ power to detect a $20 \%$ relative risk reduction in the primary renal endpoint based on events being observed in 844 patients, and a 2 -sided $p$ value of 0.05 . A $20 \%$ relative risk reduction is considered to be a clinically meaningful effect commensurate with the risk reductions seen in studies of agents targeting the renin-angiotensin-aldosterone blockade [5, 6]. Assumptions include an event rate in the placebo arm of $6.5 \%$ per year (similar to the rate seen in the placebo arm of the Aliskiren Trial in Type 2 Diabetes Using Cardio-Renal Endpoints [ALTITUDE] study [17]), a premature discontinuation rate of $6 \%$ per year, $1 \%$ loss to follow-up, an enrollment duration of 27 months, and a total study duration of approximately 60 months.

An interim analysis will also be conducted by the Independent Data Monitoring Committee (IDMC) when primary efficacy events have been observed in 405 patients. The alpha spending function will be used, and the alpha spent to test the primary composite endpoint in the interim analysis is 0.01 . If the conditional power (based on the assumption that the hazard ratio in the remaining study is 0.80 ) is $\leq 10 \%$, the study may be stopped for futility at the time of the interim analysis.

Ethics

Regulatory approval for the conduct of the trial is obtained in each country, and ethics approval, at every site.

\section{Study Oversight}

The CREDENCE Steering Committee is comprised of 17 independent academics and 2 representatives of the trial sponsor. The Steering Committee has scientific responsibility for the design, conduct, data collection, analysis, and reporting of the trial. Dayto-day trial management is provided by a Contract Research Organization, an Academic Research Organization, and the sponsor under the oversight of the Steering Committee. The Steering Committee will review study progress, will have full access to the trial database after trial completion, will conduct independent analysis of the main outcomes according to a prespecified Statistical Analysis Plan, and will be responsible for final publication decisions. An IDMC is responsible for reviewing safety in an unblinded manner. In addition to reviewing safety during the study, the IDMC will also review the interim analysis results among other parameters. A description of the roles and responsibilities of each of these entities is shown in online supplementary Appendix 4.

\section{Current Status}

The study recruited 4,401 participants from 34 countries between March 2014 and May 2017. Baseline characteristics for the enrolled patients are shown in Table2. Of the 4,401 patients enrolled, $66 \%$ were men and the mean age was 63.0 years. Patients had a mean duration of T2DM of 15.8 years and $\mathrm{HbA} 1 \mathrm{c}$ of $8.3 \%$. Baseline mean eGFR was $56.2 \mathrm{~mL} / \mathrm{min} / 1.73 \mathrm{~m}^{2}$ and median UACR was $927 \mathrm{mg} / \mathrm{g}$.

\section{Discussion}

CREDENCE will rigorously assess whether the SGLT2 inhibitor canagliflozin reduces the risk of a composite kidney and cardiovascular outcome in individuals with diabetes at high risk of progression of CKD. The glucoselowering efficacy of canagliflozin and other SGLT2 inhibitors has been shown to be diminished in individuals with T2DM and CKD due to the renal mechanism of action; however, the favorable effects of agents in this class on multiple parameters other than glucose, including BP, weight, and albuminuria, may contribute to improvements in renal outcomes in this population, as these parameters have been associated with reduced risk of kidney 
Table 2. Baseline demographic and disease characteristics

\begin{tabular}{lc}
\hline Characteristic & Total $(n=4,401)$ \\
\hline Gender, $n(\%)$ & \\
$\quad$ Male & $2,907(66.1)$ \\
$\quad$ Female & $1,494(33.9)$ \\
Age, years & $63.0 \pm 9.2$ \\
Race, $n(\%)$ & \\
$\quad$ White & $2,932(66.6)$ \\
$\quad$ Black or African American & $223(5.1)$ \\
$\quad$ Asian & $877(19.9)$ \\
$\quad$ Other* & $369(8.4)$ \\
BMI, kg/m ${ }^{2}$ & $31.3 \pm 6.2$ \\
HbA1c, $\%$ & $8.3 \pm 1.3$ \\
Duration of T2DM, years & $15.8 \pm 8.7$ \\
eGFR, mL/min/1.73 m ${ }^{2}$ & $56.2 \pm 18.2$ \\
Median UACR, mg/g & 927.0 \\
Systolic BP, mmHg & $140.0 \pm 15.6$ \\
Diastolic BP, mmHg & $78.3 \pm 9.4$ \\
LDL-C, mmol/L & $2.5 \pm 1.1$ \\
\hline
\end{tabular}

BMI, body mass index; T2DM, type 2 diabetes mellitus; HbA1c, glycated hemoglobin; eGFR, estimated glomerular filtration rate; UACR, urinary albumin:creatinine ratio; BP, blood pressure; LDL$\mathrm{C}$, low-density lipoprotein cholesterol. Data are mean \pm SD unless otherwise indicated.

* Includes American Indian or Alaska Native, Native Hawaiian or other Pacific Islander, multiple, other, unknown, or not reported.

disease in some studies [18,19]. The effects of canagliflozin on intra-renal hemodynamics are likely an important contributor to the beneficial effects on kidney function [20]. This may occur as part of the signaling cascade stemming from the blockade of SGLT2 in the proximal tubule, which may alter sodium reabsorption and atrial natriuretic peptide release $[21,22]$. A reduction in intraglomerular pressure manifests clinically as an acute reduction in GFR and lower albuminuria, both of which have been demonstrated to occur with canagliflozin $[13,18]$.

CREDENCE will rigorously and reliably assess the effects of canagliflozin on a composite kidney outcome utilizing components that have been commonly used in previous trials. The inclusion of ESKD requiring dialysis or transplantation has been universal in kidney outcome trials. One important nuance in CREDENCE is that the composite outcome includes patients with an eGFR level that falls persistently below $15 \mathrm{~mL} / \mathrm{min} / 1.73 \mathrm{~m}^{2}$. The component was chosen, as it is believed to be clinically important, given the high rates of morbidity and ill health seen below this level of kidney function, and because it reduces some of the subjectivity seen in the timing of di- alysis commencement [23]. Doubling of creatinine was chosen in contrast to lesser changes in kidney function (e.g., 40 or $50 \%$ reductions in eGFR) due to previous data showing that canagliflozin causes an acute reduction in eGFR, which increases the risk of a type I error for these lesser reductions as compared to doubling in this context [24]. Finally, death due to ESKD or cardiovascular disease was included: (i) to capture similar expected effects on these causes of mortality, (ii) because effects on death due to other causes are not expected, and (iii) because these causes of death share many common risk factors and pathways and may be difficult to differentiate.

In providing definitive evidence on the impact of canagliflozin on kidney and cardiovascular outcomes for individuals with substantive kidney disease, CREDENCE complements other large outcome trials designed to generate evidence on the cardiovascular safety of SGLT2 inhibition in individuals with T2DM and mild or no kidney disease (Fig. 2). These trials include the completed Empagliflozin Cardiovascular Outcome Event Trial in T2DM Patients (EMPA-REG OUTCOME; NCT01131676) [14] and an integrated analysis of data from the CANagliflozin cardioVascular Assessment Study (CANVAS; NCT01032629) and CANVAS-R (renal endpoints; NCT01989754), which comprise the CANVAS Program [25]. Data supporting the potential benefits on kidney outcomes with SGLT2 inhibitors have already emerged from secondary analyses of the EMPA-REG OUTCOME trial $[14,15]$. In addition, the CANVAS Program has recently reported effects on progression of albuminuria and a composite renal endpoint [16]. Thus, there may be additional value in pooling data from CREDENCE and the CANVAS Program to gain further insights on the renal and cardiovascular effects of canagliflozin.

In contrast to the EMPA-REG OUTCOME and CANVAS Program cardiovascular outcomes trials, CREDENCE has been designed to specifically assess effects on clinically important outcomes in individuals with diabetes at high risk of kidney progression (Table 3 ). CKD has been associated with an increased risk of cardiovascular disease [26, 27], so the inclusion of an enriched population of patients with CKD in CREDENCE will, by extension, also include a large number of people with a high risk of cardiovascular disease. One important distinction is that the primary outcome in CREDENCE is a composite of renal and cardiovascular endpoints. Consequently, patients were required to be on a stable regimen of the maximum tolerated dose of ACEi or ARB, which will help address the role of SGLT2 inhibition within the 


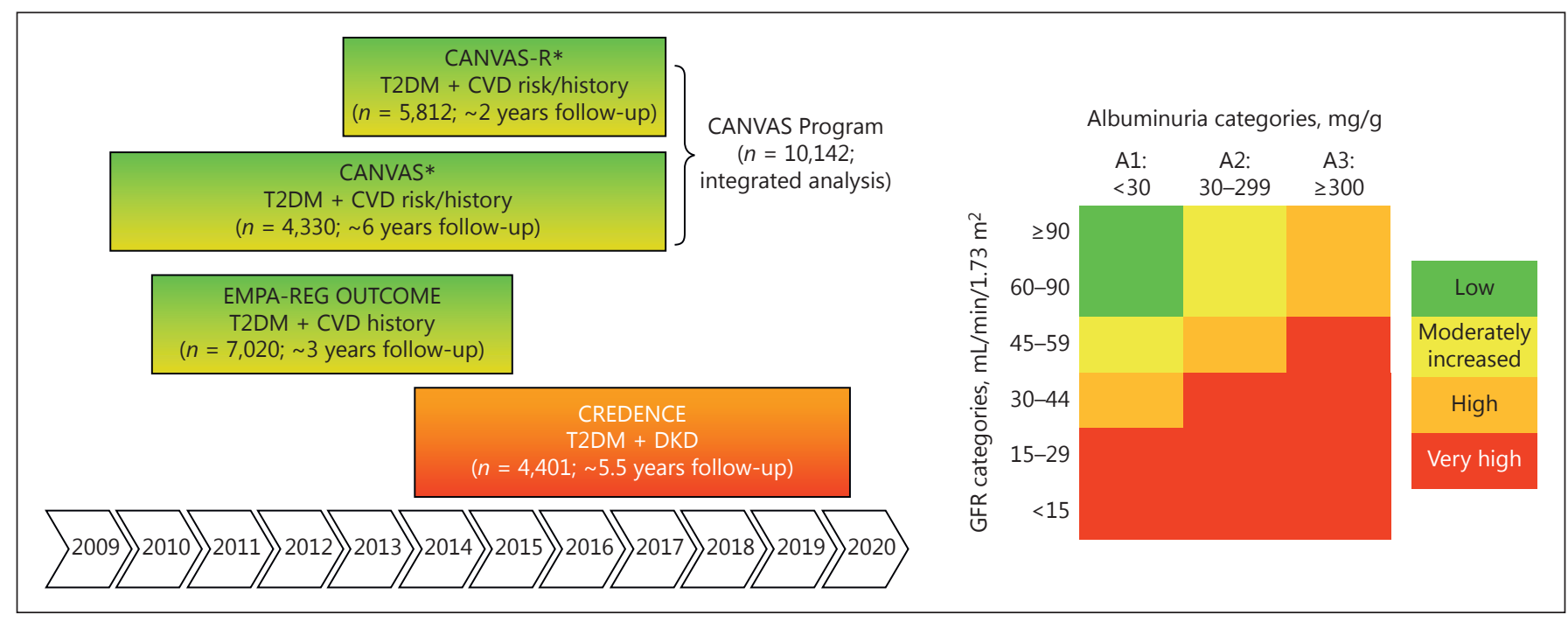

Fig. 2. Overview of trial timelines. CANVAS, CANagliflozin cardioVascular Assessment Study; T2DM, type 2 diabetes mellitus; CVD, cardiovascular disease; CANVAS-R, CANagliflozin cardioVascular Assessment Study-Renal; EMPA-REG, Empagliflozin Cardiovascular Outcome Event Trial in Type 2 Diabetes Mellitus Patients;
CREDENCE, Canagliflozin and Renal Events in Diabetes with Established Nephropathy Clinical Evaluation; DKD, diabetic kidney disease; GFR, glomerular filtration rate; CKD, chronic kidney disease. ${ }^{*}$ Note that the patient populations in CANVAS and CANVAS$\mathrm{R}$ are nearly identical to facilitate an integrated analysis of the data. current standard of care for renal disease. Additionally, the study population in CREDENCE is at an increased risk of kidney events and the planned trial duration should be sufficiently long to observe renal-related events. Finally, all primary renal outcomes in CREDENCE will be adjudicated and doubling of serum creatinine will be confirmed with a repeat specimen to assess whether changes were sustained.

CREDENCE will also accumulate additional data on specific safety risks that have been associated with canagliflozin treatment, such as diabetic ketoacidosis [9], fracture [10], amputation [11], and acute kidney injury [12] that will enhance the understanding of the balance of risks and benefits associated with use of canagliflozin in patients with reduced kidney function who may be more susceptible to adverse effects of treatment.

In conclusion, the existing evidence base for canagliflozin and other SGLT2 inhibitors in patients with normal or mildly impaired renal function supports a potential benefit for these therapies on renal and cardiovascular outcomes in patients with impaired kidney function. CREDENCE is specifically designed to assess the effects of canagliflozin on renal and cardiovascular outcomes in patients with T2DM at high risk of kidney disease and provide definitive evidence for the effect of SGLT2 inhibition in this population. If the promising preliminary data are confirmed, canagliflozin could lead to a substantial reduction in the global burden of kidney failure due to T2DM.

\section{Acknowledgments}

Steering Committee: Vlado Perkovic (Chair), Kenneth W. Mahaffey (co-chair), Rajiv Agarwal, George Bakris, Barry M. Brenner, Christopher P. Cannon, David M. Charytan, Dick de Zeeuw, Tom Greene, Meg J. Jardine, Hiddo J.L. Heerspink, Adeera Levin, Bruce Neal, Carol Pollock, David C. Wheeler, Hong Zhang, Bernard Zinman.

Independent Data Monitoring Committee: Darren Maguire (chair), Rury Holman, Philip Home, Dan Scharfstein, Patrick Parfrey.

Endpoint Adjudication Committee: Rajiv Agarwal (chair), Kenneth W. Mahaffey (co-chair), Shahnaz Shahinfar, Phyllis August, Tara Chang, Arjun D. Sinha, James Januzzi, Daniel Kolansky, John Amerena, Graham Hillis, Philip Gorelick, Brett Kissela, Scott Kasner, Richard Lindley, Greg Fulcher.

Sponsor: This study is sponsored by Janssen Research and Development, LLC. The sponsor was involved in the study design, the writing of the report, and the decision to submit the article for publication.

Collaborating Sites and Investigators: See online supplementary Appendix 1.

National Lead Investigators: Diego Aizenberg (Argentina and Chile), Roberto Pecoits Filho (Brazil), Adeera Levin and David Cherney (Canada), Gregorio Obrador (Mexico, Colombia, and Guatemala), Glen Chertow and Tara Chang (United States), Car- 
Table 3. Key design differences among large clinical studies of SGLT2 inhibition in T2DM

\begin{tabular}{|c|c|c|c|}
\hline Population specifics & CREDENCE & CANVAS Program [25] & EMPA-REG OUTCOME [14] \\
\hline eGFR & $\begin{array}{l}\geq 30 \text { to }<90 \mathrm{~mL} / \mathrm{min} / 1.73 \mathrm{~m}^{2} \\
\text { (with target of } \sim 60 \% \text { with eGFR } 30-<60 \text { ) } \\
\text { (CKD-EPI) }\end{array}$ & $\geq 30 \mathrm{~mL} / \mathrm{min} / 1.73 \mathrm{~m}^{2}(\mathrm{MDRD})$ & $\geq 30 \mathrm{~mL} / \mathrm{min} / 1.73 \mathrm{~m}^{2}(\mathrm{MDRD})$ \\
\hline UACR & $>300$ to $\leq 5,000 \mathrm{mg} / \mathrm{g}$ & No criteria & No criteria \\
\hline $\mathrm{HbAlc}$ & $\geq 6.5$ to $\leq 12.0 \%$ & $\geq 7.0$ to $\leq 10.5 \%$ & $\begin{array}{l}\text { Drug-naïve: } \geq 7.0 \text { to } \leq 9.0 \% \\
\text { Subjects on stable AHA: } \geq 7.0 \text { to } \leq 10.0 \%\end{array}$ \\
\hline ACEi or ARB usage at enrollment & $\begin{array}{l}\text { Mandatory (maximum tolerated } \\
\text { labeled dose stable at least } 4 \text { weeks } \\
\text { prior to randomization) }\end{array}$ & $\begin{array}{l}\text { No criteria (other than standard of care based on } \\
\text { local practice) }\end{array}$ & No criteria \\
\hline CV history inclusion & No requirement & $\begin{array}{l}\geq 30 \text { years with a history of symptomatic } \\
\text { atherosclerotic coronary, cerebrovascular, or } \\
\text { peripheral vascular disease, or } \geq 50 \text { years with } \geq 2 \\
\text { vascular disease risk factors (diabetes duration } \geq 10 \\
\text { years, systolic } \mathrm{BP}>140 \mathrm{mmHg} \text { on } \geq 1 \text { BP-lowering } \\
\text { agent, current smoker, albuminuria, or HDL-C } \\
<1 \mathrm{mmol} / \mathrm{L} \text { ) }\end{array}$ & $\begin{array}{l}\text { Established or high risk of CV events } \\
\text { (History of } \mathrm{MI} \text {, stroke, and/or unstable angina } \\
>2 \text { months prior to participation, and/or } \\
\text { multi-vessel CAD, left main CAD, single-vessel } \\
\text { CAD, and/or occlusive PAD) }\end{array}$ \\
\hline Design & Placebo-controlled trial & Placebo-controlled trials & Placebo-controlled trial \\
\hline Enrollment & 4,401 & $\begin{array}{l}10,142 \\
\text { (includes 4,330 in CANVAS, 5,812 in CANVAS-R) }\end{array}$ & 7,020 \\
\hline Agent & $\begin{array}{l}100 \mathrm{mg} \text { canagliflozin or matching } \\
\text { placebo }\end{array}$ & $\begin{array}{l}100 \mathrm{mg} \text { canagliflozin, } 300 \mathrm{mg} \text { canagliflozin, or } \\
\text { matching placebo (in CANVAS-R } 100 \mathrm{mg} \\
\text { uptitrated to } 300 \mathrm{mg} \text { or matching placebo) }\end{array}$ & $\begin{array}{l}10 \mathrm{mg} \text { empagliflozin, } 25 \mathrm{mg} \text { empagliflozin, or } \\
\text { matching placebo }\end{array}$ \\
\hline Expected duration & Mean $\sim 5.5$ years & $\begin{array}{l}\text { Mean } 5.7 \text { years (CANVAS), and } \\
\text { Mean } 2.1 \text { years (CANVAS-R)* }\end{array}$ & Median 3.1 years* \\
\hline Primary endpoint & $\begin{array}{l}\text { Composite of ESKD, doubling of serum } \\
\text { creatinine, and renal or CV death }\end{array}$ & $\begin{array}{l}\text { Composite of CV death, nonfatal MI, and } \\
\text { nonfatal stroke }\end{array}$ & $\begin{array}{l}\text { Composite of CV death, nonfatal MI, and } \\
\text { nonfatal stroke }\end{array}$ \\
\hline $\begin{array}{l}\text { Prespecified renal clinical } \\
\text { endpoints }\end{array}$ & $\begin{array}{l}\text { Composite of ESKD, doubling of serum } \\
\text { creatinine, and renal death } \\
\text { ESKD } \\
\text { Doubling of serum creatinine } \\
\text { Renal death }\end{array}$ & $\begin{array}{l}\text { Composite of } 40 \% \text { reduction in eGFR, renal } \\
\text { death, or RRT } \\
\text { Composite of } 40 \% \text { reduction in eGFR, renal death, } \\
\text { RRT, or CV death } \\
\text { Composite of } 40 \% \text { reduction in eGFR, } \\
\text { macroalbuminuria, renal death, or RRT } \\
\text { Composite of doubling of serum creatinine, renal } \\
\text { death, or RRT } \\
\text { Composite of doubling of serum creatinine, renal } \\
\text { death, RRT, or CV death } \\
\text { Composite of doubling of serum creatinine, } \\
\text { macroalbuminuria, renal death, or RRT }\end{array}$ & $\begin{array}{l}\text { Composite microvascular outcome, which } \\
\text { included new/worsening nephropathy }{ }^{\dagger} \\
\text { Composite new/worsening nephropathy and } \\
\text { CV death } \\
\text { Doubling of creatinine with eGFR } \leq 45 \mathrm{~mL} / \\
\mathrm{min} / 1.73 \mathrm{~m}^{2}\end{array}$ \\
\hline Post hoc renal clinical endpoints & None & None & $\begin{array}{l}\text { Composite of doubling of serum creatinine with } \\
\text { eGFR } \leq 45 \mathrm{~mL} / \mathrm{min} / 1.73 \mathrm{~m}^{2} \text {, initiation of RRT, } \\
\text { or death due to renal disease }\end{array}$ \\
\hline $\begin{array}{l}\text { Confirmation of doubling of } \\
\text { serum creatinine }\end{array}$ & $\begin{array}{l}\text { Doubling of serum creatinine } \\
\text { confirmed on repeat specimen after } \\
30 \text { days unless circumstances prevent } \\
\text { this (e.g., death or dialysis) or if there } \\
\text { is clear evidence that the doubling is } \\
\text { related to an acute kidney injury }\end{array}$ & $\begin{array}{l}\text { Doubling of serum creatinine confirmed on repeat } \\
\text { specimen after } 30 \text { days unless circumstances } \\
\text { prevent this (e.g., death or dialysis) or if there is } \\
\text { clear evidence that the doubling is related to an } \\
\text { acute kidney injury }\end{array}$ & No confirmation \\
\hline Renal biochemical endpoints & $\begin{array}{l}\text { Changes in eGFR over time } \\
\text { Changes in albuminuria over time }\end{array}$ & $\begin{array}{l}\text { Progression of albuminuria } \\
\text { Regression of albuminuria } \\
\text { eGFR changes over time } \\
\text { Changes in UACR over time }\end{array}$ & $\begin{array}{l}\text { New-onset albuminuria (UACR } \geq 30 \mathrm{mg} / \mathrm{g} \text { ) } \\
\text { New-onset macroalbuminuria (UACR } \\
\geq 300 \mathrm{mg} / \mathrm{g} \text { ) }\end{array}$ \\
\hline
\end{tabular}

SGLT2, sodium glucose co-transporter 2; T2DM, type 2 diabetes mellitus; CREDENCE, Canagliflozin and Renal Events in Diabetes with Established Nephropathy Clinical Evaluation; CANVAS, CANagliflozin cardioVascular Assessment Study; EMPA REG OUTCOME, Empagliflozin Cardiovascular Outcome Event Trial in Type 2 Diabetes Mellitus Patients; eGFR, estimated glomerular filtration rate; CKD-EPI, chronic kidney isease epidemiologic collaboration; MDRD, Modification of Diet in Renal Disease; UACR, urinary albumin:creatinine ratio; HbAlc, glycated hemoglobin; $\mathrm{AHA}$, antihyperglycemic agent; $\mathrm{ACEi}$, angiotensin-converting enzyme inhibitor; ARB, angiotensin receptor blocker; CV, cardiovascular; BP, blood pressure; HDL-C, high-density lipoprotein cholesterol; MI, myocardial infarction; CAD, coronary artery disease; PAD, peripheral artery disease; ESKD, endstage kidney disease; RRT, renal replacement therapy.

* Actual follow-up periods.

${ }^{\dagger}$ New or worsening nephropathy defined in EMPA-REG OUTCOME as new-onset macroalbuminuria, doubling of serum creatinine with eGFR $\leq 45 \mathrm{~mL} / \mathrm{min} / 1.73 \mathrm{~m}{ }^{2}$, initiation of RRT, or death due to renal disease. 
mel Hawley (Australia and New Zealand), Linong Ji and Hong Zhang (China), Takashi Wada (Japan), Vivekanand Jha (India), Soo Kun Lim (Malaysia), Florence Santos (Philippines), DongWan Chae (South Korea), Shang-Jyh Hwang (Taiwan), Evgueniy Vazelov (Bulgaria), Ivan Rychlik (Czech Republic and Slovakia), Samy Hadjadj (France), Vera Krane (Germany), Laszlo Rosivall (Hungary), Luca De Nicola (Italy), Alexander Dreval (Lithuania and Russia), Michal Nowicki (Poland), Adalbert Schiller (Romania), Larry Distiller (South Africa), Jose Luis Gorriz (Spain), Mykola Kolesnyk (Ukraine), David Wheeler (United Kingdom).

\section{Disclosure Statement}

All of the authors received research support or consulting fees from Janssen in relation to their roles on the steering committee of the CREDENCE trial.

M.J.J. is supported by a co-funded National Health and Medical Research Council Career Development Fellowship and National Heart Foundation Future Leader Fellowship; is responsible for research projects that have received unrestricted funding from Gambro, Baxter, CSL, Amgen, Eli Lilly, and Merck; has served on advisory boards and/or spoken at scientific meetings sponsored by Boehringer Ingelheim, Baxter, Amgen and Roche; and directs honoraria to clinical research programs. K.W.M.'s financial disclosures after August 1, 2013, can be viewed at http://med.stanford. edu/profiles/kenneth-mahaffey. B.N. is supported by an Australian National Health and Medical Research Council Principal Research Fellowship; holds a research grant for this study from Janssen; and has held research grants for other large-scale cardiovascular outcome trials from Roche, Servier and Merck Schering Plough; and his institution has received consultancy, honoraria, or travel support for contributions he has made to advisory boards and/or the continuing medical education programs of Abbott, Janssen, Novartis, Pfizer, Roche, and Servier. R.A. has received personal fees from Bayer, Johnson \& Johnson, Boehringer Ingelheim, Takeda, Daiichi Sankyo, Amgen, Celgene, and Eli Lilly during the conduct of the study. G.L.B. has received research funding paid to the University of Chicago for serving as principal investigator on national clinical trials for Bayer, Janssen, and AbbVie; has served as a consultant for Merck, Vascular Dynamics, and Relypsa; has served as Editor-in-Chief of the American Journal of Nephrology, as the Nephrology and Hypertension Section Editor of UpToDate, and as an associate editor of Diabetes Care, Hypertension Research, and Nephrology Dialysis and Transplantation. B.M.B. has no pertinent disclosures aside from his involvement in this trial. S.B., R.E., J.X., and M.D. are full-time employees of Janssen Research and Development, LLC. C.P.C. has received research grants (all >USD 10,000) from Amgen, Boehringer Ingelheim, Bristol-Myers Squibb, Daiichi Sankyo, and Arisaph and Takeda; and has received consulting fees from Alnylam, Amgen, Arisaph, Astra Zeneca, Boehringer Ingelheim, Bristol-Myers Squibb, GlaxoSmithKline, Kowa, Lipimedix*, Merck, Pfizer, Regeneron*, Sanofi*, and Takeda (* denotes $>$ USD 10,000). D.M.C. is a consultant for Medtronic, Zoll, PLC Medical, and Eli Lilly. D.d.Z. is a consultant for and received honoraria (to employer) from AbbVie, Astellas, Boehringer Ingelheim, Bayer, Eli Lilly, Fresenius, Janssen, and Merck. T.G. has received consulting fees from Janssen, Durect, and Pfizer. H.J.L.H. has served as a consultant for Abbvie Astellas,
AstraZeneca, Boehringer Ingelheim, Fresenius, Janssen, and Merck and has received grant support from AstraZeneca and Boehringer Ingelheim; he has a policy that all honoraria are paid to his institution, University Medical Center Groningen. A.L. has no disclosures. C.P. is a member of the CREDENCE steering committee and has received honoraria for serving on advisory boards and as a speaker for Merck Sharpe and Dohme, AstraZeneca, Boehringer Ingelheim/Eli Lilly. D.C.W. has received honoraria from Janssen to compensate for time spent working on the trial steering committee and has received speaker fees from Janssen. H.Z. has no competing interests to declare. B.Z. has served as a consultant and received honoraria from AstraZeneca, Boehringer Ingelheim, Eli Lilly, Janssen, MSD, Novo Nordisk, and Sanofi and has received grant support from Boehringer Ingelheim, Novo Nordisk, and AstraZeneca. V.P. is supported by a Senior Research Fellowship from the Australian National Health and Medical Research Council; has served on advisory boards and/or spoken at scientific meetings sponsored by Janssen, Baxter, AbbVie, Astellas, Boehringer Ingelheim, AstraZeneca, Eli Lilly, Merck, and GlaxoSmithKline; and has a policy of honoraria going to his employer.

\section{Funding Sources}

This trial is sponsored by Janssen Research and Development, LLC. Medical writing support was sponsored by Janssen Global Services, LLC.

\section{Author Contribution}

The Steering Committee designed the study in conjunction with the Sponsor. M.J.J. wrote the first draft of the paper, had full access to the study design information, and had final responsibility for the decision to submit for publication. All authors provided input for the preparation of subsequent drafts and approved the final version for submission. Technical editorial assistance was provided by Kimberly Dittmar, $\mathrm{PhD}$, of MedErgy, and was funded by Janssen Global Services, LLC. All authors reviewed and approved the manuscript.

\section{References}

1 Liyanage T, Ninomiya T, Jha V, Neal B, Patrice HM, Okpechi I, Zhao MH, Lv J, Garg AX, Knight J, Rodgers A, Gallagher M, Kotwal S, Cass A, Perkovic V: Worldwide access to treatment for end-stage kidney disease: a systematic review. Lancet 2015;385:19751982.

2 GBD 2015 Disease and Injury Incidence and Prevalence Collaborators: Global, regional, and national incidence, prevalence, and years lived with disability for 310 diseases and injuries, 1990-2015: a systematic analysis for the Global Burden of Disease Study 2015. Lancet 2016;388:15451602.

3 International Diabetes Federation: IDF Diabetes Atlas, ed 7. Brussels, International Diabetes Federation, 2015. 
4 Astor BC, et al: Lower estimated glomerular filtration rate and higher albuminuria are associated with mortality and end-stage renal disease. A collaborative meta-analysis of kidney disease population cohorts. Kidney Int 2011;79:1331-1340.

5 Lewis EJ, Hunsicker LG, Clarke WR, Berl T, Pohl MA, Lewis JB, Ritz E, Atkins RC, Rohde $\mathrm{R}, \mathrm{Raz}$ I: Renoprotective effect of the angiotensin-receptor antagonist irbesartan in patients with nephropathy due to type 2 diabetes. N Engl J Med 2001;345:851-860.

6 Brenner BM, Cooper ME, de Zeeuw D, Keane WF, Mitch WE, Parving HH, Remuzzi G, Snapinn SM, Zhang Z, Shahinfar S: Effects of losartan on renal and cardiovascular outcomes in patients with type 2 diabetes and nephropathy. N Engl J Med 2001;345:861869.

7 Rosenthal N, Meininger G, Ways K, Polidori D, Desai M, Qiu R, Alba M, Vercruysse F, Balis D, Shaw W, Edwards R, Bull S, Di Prospero N, Sha S, Rothenberg P, Canovatchel W, Demarest K: Canagliflozin: a sodium glucose co-transporter 2 inhibitor for the treatment of type 2 diabetes mellitus. Ann N Y Acad Sci 2015; 1358:28-43.

8 Qiu R, Balis D, Xie J, Davies MJ, Desai M, Meininger G: Longer-term safety and tolerability of canagliflozin in patients with type 2 diabetes: a pooled analysis. Curr Med Res Opin 2017;33:553-562.

9 Erondu N, Desai M, Ways K, Meininger G: Diabetic ketoacidosis and related events in the canagliflozin type 2 diabetes clinical program. Diabetes Care 2015;38:1680-1686.

10 Watts NB, Bilezikian JP, Usiskin K, Edwards R, Desai M, Law G, Meininger G: Effects of canagliflozin on fracture risk in patients with type 2 diabetes mellitus. J Clin Endocrinol Metab 2016;101:157-166.

11 US Food and Drug Administration: FDA Drug Safety Communication: Interim Clinical Trial Results Find Increased Risk of Leg and Foot Amputations, Mostly Affecting the Toes, with the Diabetes Medicine Canagliflozin (Invokana, Invokamet); FDA to Investigate. http://www.fda.gov/Drugs/DrugSafety/ucm557507.htm (accessed November 9, 2017).

12 Desai M, Yavin Y, Balis D, Sun D, Xie J, Canovatchel W, Rosenthal N: Renal safety of canagliflozin, a sodium glucose co-transporter 2 inhibitor, in patients with type 2 diabetes mellitus. Diabetes Obes Metab 2017;19:897900.

13 Heerspink HJ, Desai M, Jardine M, Balis D, Meininger G, Perkovic V: Canagliflozin slows progression of renal function decline independently of glycemic effects. J Am Soc Nephrol 2017;28:368-375.

14 Zinman B, Wanner C, Lachin JM, Fitchett D, Bluhmki E, Hantel S, Mattheus M, Devins T, Johansen OE, Woerle HJ, Broedl UC, Inzucchi SE: Empagliflozin, cardiovascular outcomes, and mortality in type 2 diabetes. N Engl J Med 2015;373:2117-2128.

15 Wanner C, Inzucchi SE, Lachin JM, Fitchett $D$, von Eynatten $M$, Mattheus $M$, Johansen OE, Woerle HJ, Broedl UC, Zinman B: Empagliflozin and progression of kidney disease in type 2 diabetes. N Engl J Med 2016;375:323334.

16 Neal B, Perkovic V, Mahaffey KW, de Zeeuw D, Fulcher G, Erondu N, Shaw W, Law G, Desai M, Matthews D; CANVAS Program Collaborative Group: Canagliflozin and cardiovascular and renal events in type 2 diabetes. N Engl J Med 2017;377:644-657.

17 Heerspink HJ, Persson F, Brenner BM, Chaturvedi N, Brunel P, McMurray JJ, Desai AS, Solomon SD, Pfeffer MA, Parving HH, de Zeeuw D: Renal outcomes with aliskiren in patients with type 2 diabetes: a prespecified secondary analysis of the ALTITUDE randomised controlled trial. Lancet Diabetes Endocrinol 2016;4:309-317.

18 Perkovic V, Heerspink HL, Chalmers J, Woodward M, Jun M, Li Q, MacMahon S, Cooper ME, Hamet P, Marre M, Mogensen CE, Poulter N, Mancia G, Cass A, Patel A, Zoungas S: Intensive glucose control improves kidney outcomes in patients with type 2 diabetes. Kidney Int 2013;83:517-523.

19 Lv J, Ehteshami P, Sarnak MJ, Tighiouart H, Jun M, Ninomiya T, Foote C, Rodgers A, Zhang H, Wang H, Strippoli GF, Perkovic V: Effects of intensive blood pressure lowering on the progression of chronic kidney disease: a systematic review and meta-analysis. CMAJ 2013;185:949-957.

20 Cherney DZ, Perkins BA, Soleymanlou N, Maione M, Lai V, Lee A, Fagan NM, Woerle HJ, Johansen OE, Broedl UC, von Eynatten M: Renal hemodynamic effect of sodium-glucose cotransporter 2 inhibition in patients with type 1 diabetes mellitus. Circulation 2014;129:587-597.
21 Ortola FV, Ballermann BJ, Anderson S, Mendez RE, Brenner BM: Elevated plasma atrial natriuretic peptide levels in diabetic rats. Potential mediator of hyperfiltration. J Clin Invest 1987;80:670-674.

22 Ballermann BJ, Brenner BM: Atrial natriuretic peptide and the kidney. Am J Kidney Dis 1987;10:7-12.

23 Lambers Heerspink HJ, Weldegiorgis M, Inker LA, Gansevoort R, Parving HH, Dwyer JP, Mondal H, Coresh J, Greene T, Levey AS, de Zeeuw D: Estimated GFR decline as a surrogate end point for kidney failure: a post hoc analysis from the Reduction of End Points in Non-Insulin-Dependent Diabetes With the Angiotensin II Antagonist Losartan (RENAAL) study and Irbesartan Diabetic Nephropathy Trial (IDNT). Am J Kidney Dis 2014;63:244-250.

24 Inker LA, Lambers Heerspink HJ, Mondal H, Schmid CH, Tighiouart H, Noubary F, Coresh J, Greene T, Levey AS: GFR decline as an alternative end point to kidney failure in clinical trials: a meta-analysis of treatment effects from 37 randomized trials. Am J Kidney Dis 2014;64:848-859.

25 Neal B, Perkovic V, Mahaffey KW, Fulcher G, Erondu N, Desai M, Shaw W, Law G, Walton MK, Rosenthal N, de Zeeuw D, Matthews DR: Optimizing the analysis strategy for the CANVAS program-a pre-specified plan for the integrated analyses of the CANVAS and CANVAS-R trials. Diabetes Obes Metab 2017;19: 926-935.

26 Herzog CA, Asinger RW, Berger AK, Charytan DM, Díez J, Hart RG, Eckardt KU, Kasiske BL, McCullough PA, Passman RS, DeLoach SS, Pun PH, Ritz E: Cardiovascular disease in chronic kidney disease. A clinical update from kidney disease: improving global outcomes (KDIGO). Kidney Int 2011;80:572586

27 Matsushita K, Coresh J, Sang Y, Chalmers J, Fox C, Guallar E, Jafar T, Jassal SK, Landman GW, Muntner P, Roderick P, Sairenchi T, Schottker B, Shankar A, Shlipak M, Tonelli M, Townend J, van Zuilen A, Yamagishi K, Yamashita K, Gansevoort R, Sarnak M, Warnock DG, Woodward M, Arnlov J; CKD Prognosis Consortium: Estimated glomerular filtration rate and albuminuria for prediction of cardiovascular outcomes: a collaborative meta-analysis of individual participant data. Lancet Diabetes Endocrinol 2015;3:514-525. 